\title{
Nebulizaciones de fenoterol-ipratropio en lactantes con síndrome bronquial obstructivo
}

\author{
M.C. Mario Calro G. ${ }^{1}$; M.C. Karin Grob $\mathbf{B}{ }^{2}$; E.U. Marion Hertmann $\mathbf{H}^{2}{ }^{2}$; Bioest. Francisco Marin $\mathbf{M}^{3}$
}

\author{
Inhaled fenoterol-ipratropium mixture in infants with acute \\ obstructive bronchial symptoms
}

\begin{abstract}
A double blina prospective study was done in 120 infants with acute obstructive bronchial disease. One half of them received combined fenoterol-ipratropium solution by inhalation in three different dose schedules while the other half was given inhalatory normal saline solution for comparative purposes. Statistical analysis showed a significant difference in favor of fenoterol-ipratropium treatment over controls as to severity of bronchial obstruction, days of recovery and the need of additional bronchodilator drugs. However, no significant differences in the response of these parameters were noted among patients receiving different dose schedules of fenoterolipratropium. The combination of fenoterol-jpratropium showed a beneficial bronchoditator effect in patients with ecute obstructive bronchial disease.

(Key words: asthma, bronchial spasm, bronchodilator agents, infant.)

La efectividad de los broncodilatadores en el período de lactantes continúa siendo un tema contradictorio $^{1-3}$. En el último tiempo se han publicado nuevos elementos clínicos y de laboratorio, apoyando el uso de broncodilatadores en el menor de 2 años, sean $\beta-2$ adrenérgicos 0 , en

1. Instituto de Pediatría, Facultad de Medicina, Universidad Austral de Chile.

2. Servicio de Pediatría, Hospital Reglonal de Valdivia.

3. Bioestadístico, Instituto de Salud Pública, Facultad de Medicina, Universidad Austral de Chile.

especial, anticolinérgicos derivados de la atropina como el bromuro de ipratropio ${ }^{4-8}$.

Por otro lado, el tratamiento local del síndrome obstructivo ha demostrado ser más efectivo que el mismo medicamento por vía oral o parenteral, al conseguir concentraciones muy superiores en el tejido pulmonar con menores efectos secundarios. A su vez, la lenta absorción de los aerosoles por las mucosas de las vias respiratorias produce un excelente efecto terapéutico, ya que éste es uniforme y de larga duración ${ }^{9-14}$.

En pediatría la utilización de aerosoles presurizados o inhaletas está limitada en lactantes y
\end{abstract}


niños menores. En ellos la utilización de nebulizadores es el tratamiento ideal por no requerir la cooperación y coordinación del paciente ${ }^{15-17}$.

En el presente trabajo deseamos evaluar el efecto broncodilatador de la combinación feno. terol-ipratropio ${ }^{18}$ en nebulizaciones en lactantes con sindrome bronquial obstructivo agudo.

\section{MATERLAL Y METODO}

El estudio se realizó de modo prospectivo en 120 lactantes con síndrome bronquial obstructjvo agudo, que por su intensidad requerian hospitalización en la Unidad de Lactantes del Hospital Regional de Valdivia, en el período de abril de 1988 a mayo de 1989. Los pacientes se distribuyeron secuencialmente en 6 grupos en sistema doble ciego, 20 lactantes por grupo. Los grupos 1,3 y 5 recibieron inhalaciones de fenoterolipratropio $v$ los grupos 2, 4 y 6 solución de cloruro te sodio 9 \%o $_{0}$, en las siguientes dosis:

Grupos 1 y 2.1 a 6 meses de edad $0,1 \mathrm{ml} \cdot d o s i s ; 6$ a 12 meses de edad 0,2 ml-dosis; 12 a 18 meses de edad 0,3 $\mathrm{ml}$-dosis y 18 a 24 meses de edad $0,4 \mathrm{ml} \cdot$ dosis. Inhalaciones cada 8 horas.

Grupos 3 y 4. 0,1 ml.kg dosis: máximo $1 \mathrm{ml} \cdot d o s i s$ Inhalaciones cada 8 horas.

Grupos 5 y 6. Igual dosis que grupos 1 y 2 pero inhalaciones cada 12 hotas. Todas estas dosis se agregan a 2 ml de solución $\mathrm{NaCl} 9 \%$, que los niños inhalaron hasta consumir toda la mezcla, lo cual ocurrió habitualmente en 10 minutos.

Un ml (20 gotas) de los medicamentos para inhalar contiene bromuro de jpratropio $0,25 \mathrm{mg}$ y bromhidrato de fenoterol $0,5 \mathrm{mg}$. La terapia inhalatoria se realizó mediante un compresor portátil, modelo Mirage; M.A.P. (Italia).
Se confeccionó una ficha estándar para registrar edad, sexo, estado de nutrición, diagnóstico, frecuencia respiratoria y cardiaca, retracción de partes blandas, tos, disminución de murmullo vesicular, sibilancias, cianosis, uso de antibióticos, necesidad de otro(s) broncodilatador(es) o corticoides y eventuales efectos secundarios indeseables como taquicardia, enrojecimiento facial, temblor, vómitos, sequedad de mucosas y excitación psicomotora en todos los pacientes.

Se empleó la escala de Bierman y Pierson modificada por Tal para calificar la severidad de la obstrucción ${ }^{19}$. Se consideró que la obstrucción bronquial era leve entre 0 y 4 puntos, moderada entre 5 y 8 , grave entre 9 y 12 puntos.

En los casos en que se mantuvo la puntuación de ingreso, transcurridas 24 horas de tratamiento o ta obstrucción bronquial aumentó a la categoría de grave, se calificó la evolución clínica como desfavorable, agtegándose tratamiento con salbutamol oral y corticoides por vía parenteral en los casos más graves. Asimismo, se utilizaron antibióticos en aquellos niños en que, por signos clínicos, de laboratorio o anbos, se sospechó una infección bacteriana, manteniéndose a estos últimos pacientes en sus respectivos grupos.

Los datos se procesaron mediante computador. La significación estadistica se determinó mediante dócima $\mathrm{F}$ de Fischer (ANDEVA), dócima Student-NeumannKauls (comparaciones múltiples) y análisis de varianza no paramétrico de Krustcal Wallis.

\section{RESULTADOS}

En la tabla 1 se describen las características de los pacientes estudiados, no existiendo diferencias estadisticamente significativas entre ellos al comparar edad, sexo, estado de nutrición $y$ puntaje de severidad de la obstrucción al ingresar al estudio $(p>0,05)$.

Tabla 1

Características de los grupos estudiados

\begin{tabular}{|c|c|c|c|c|c|c|c|c|c|c|c|c|c|}
\hline \multirow{3}{*}{ Grupos } & \multirow{2}{*}{\multicolumn{2}{|c|}{ Edad }} & \multicolumn{4}{|c|}{ Sexo } & \multicolumn{7}{|c|}{ Estado nutritivo } \\
\hline & & & \multicolumn{2}{|c|}{ Masc. } & \multicolumn{2}{|c|}{ Fern. } & \multicolumn{2}{|c|}{$\mathbf{0}$} & \multicolumn{2}{|c|}{$\mathrm{E}$} & \multicolumn{2}{|c|}{ D1 } & \multirow{2}{*}{$\begin{array}{c}\mathrm{D} 2 \\
\mathrm{n}\left(\%_{0}\right)\end{array}$} \\
\hline & $\bar{x}$ meses & (DE) & $\mathrm{n}$ & (\%) & $\mathrm{n}$ & $(\%)$ & $n$ & $(\%)$ & n & $(\%)$ & $\pi$ & $(\%)$ & \\
\hline 1 & 9,70 & $(3,82)$ & 12 & $(60)$ & 8 & $(40)$ & 3 & (15) & 12 & (60) & 5 & (25) & $0 \quad(0)$ \\
\hline 2 & 10,20 & $(5,98)$ & 11 & (55) & 9 & (45) & 2 & (I0) & 10 & (50) & 8 & (40) & \\
\hline 3 & 9.25 & $(4,69)$ & 13 & (65) & 7 & (35) & 3 & (15) & 12 & (60) & 3 & (15) & $2(10)$ \\
\hline 4 & 10,15 & $(6,19)$ & 11 & (55) & 9 & (45) & 5 & (25) & 11 & (55) & 3 & (1s) & 1 (5) \\
\hline 5 & 9,70 & $\langle 5,78)$ & 11 & (55) & 9 & (45) & 3 & (15) & 15 & (75) & 2 & (10) & $0 \quad(0)$ \\
\hline 6 & 9,70 & $(6,55)$ & 10 & (50) & 10 & (50) & 0 & (0) & 12 & $(60)$ & 8 & (40) & $0 \quad(0)$ \\
\hline Total & 9,78 & $(5,48)$ & 68 & (57) & 52 & (43) & 16 & (13) & 72 & (60) & 29 & (24) & 3 (3) \\
\hline $\mathrm{p}$ & & $>0,05$ & & & & & & & & & & & \\
\hline
\end{tabular}


En la tabla 2 se detalla Ja evolución de la calificación del grado de obstrucción en los diferentes grupos, destacándose un comportamiento similar al interior de los controles (grupos 2, 4 y 6) y una respuesta muy favorable entre los niffos que recibieron medicamentos (grupos 1,3 y 5). Esta respuesta favorable no se relacionó con la edad de los pacientes.

Al comparar los niffos de los grupos, que recibieron medicamento, con sus respectivos controles (grupos 1 con $2 ; 3$ con $4 ;$ y 5 con 6 ), se detecta una diferencia muy significativa $(\mathrm{p}<$ 0,001 ) en favor de los grupos 1 y 3 . El grupo $S$ presento una diferencia estadísticamente significativa respecto a su control, grupo $6(p<0,05)$ el primer día, pero desde el segundo día su comportamiento fue similar a los grupos 1 y 3 ( $p<$ 0,001 ). No se encontraron diferencias significativas al comparar entre ellos a los grupos que recibieron dosis diferentes de fenoterol-ipratro. pio (tabla 3).

La necesidad de utilizar otro(s) broncodilata. dor(es) fue significativamente más alta en los grupos control (tabla 4, p <0,001). Nuevamente no hubo diferencias significativas entre los grupos que recibieron el medicamento en dosis diferentes.

El promedio general de días estada fue de $4,00(\mathrm{DE} 1,86)$ y al analizarlas entre los grupos 1 al 6 fueron 3,10 (DE 1,21); 5,00 (DE 1,69);
Tabla 3

Significación estadística de los puntajes en los grupos estudiados

\begin{tabular}{lrrrrrr}
\hline Grupos & 1 & 2 & 3 & 4 & 5 & 6 \\
\hline 1 & - & $*$ & NS & $*$ & NS & $*$ \\
2 & & - & $*$ & NS & $*$ & NS \\
3 & & & - & $*$ & NS & $*$ \\
4 & & & & - & $*$ & NS \\
5 & & & & & - & $*$ \\
6 & & & & & & - \\
\hline
\end{tabular}

* a $\mathrm{p}<0,001$.

NS $=$ No significativo $p>0,05$.

3,30 (DE 1,59); 4,95 (DE 1,73); 3,35 (DE 1,93); 4,85 (DE 1,90 ), respectivamente; con una significación estadística de $\mathrm{p}<0,001$ a favor de los grupos 1,3 y 5 que recibieron medicamento $y$ nuevamente sin diferencia significativa entre estos 3 grupos.

Con respecto a los efectos secundarios, el me. dicamento fue muy bien tolerado. No se detectó taquicardia (tabla 5), temblores, enrojecimiento facial u otro efecto indeseable en los pacientes tratados. Algunos lactantes, con medicamento o con solución salina, rechazaron inicialmente el procedimiento de nebulización, pero luego lo

Tabla 2

Califĭcación de la dificultad respiratoria de los grupos estudiados, según dias de tratamiento

\begin{tabular}{|c|c|c|c|c|c|c|c|c|c|c|c|}
\hline \multirow{2}{*}{ Grupos } & \multicolumn{2}{|l|}{ Ingreso } & Dia 1 & \multicolumn{3}{|c|}{ Día 2} & \multicolumn{2}{|l|}{ Día 3} & \multicolumn{2}{|c|}{ Día 4} & \multirow[b]{2}{*}{$\mathrm{p}$} \\
\hline & $\overline{\mathrm{x}}(\mathrm{DE})$ & $\mathrm{p}$ & $\bar{x}$ (DE) & p & $\overline{\mathrm{x}} \quad(\mathrm{DE})$ & $\mathbf{p}$ & $\bar{x}(D E)$ & p & $\overline{\mathbf{x}}$ & (DE) & \\
\hline 1 & $8,30(1,22)$ & & $5,10(1,33)$ & & $3,10(1,80)$ & & $2,33(0,89)$ & & 2,50 & $(0,84)$ & \\
\hline 2 & $8,15(1,04)$ & NS & $7,00(1,34)$ & $<0,001$ & $5,95(1,32)$ & $<0,001$ & $4,60(1,54)$ & $<0,001$ & 3,71 & $(1,31)$ & $<0,001$ \\
\hline 3 & $8,15(1,22)$ & & $5,40(1,47)$ & & $3,25(1,65)$ & & $2,36(1,82)$ & & 2,17 & $(2,40)$ & \\
\hline 4 & $7,70(1,34)$ & NS & $7,45(1,36)$ & $<0,001$ & $6,65(1,63)$ & $<0,001$ & $5,63(1,83)$ & $<0,001$ & 4,78 & $(2,05)$ & $<0,001$ \\
\hline 5 & $7,95(1,73)$ & & $6,05(2,12)$ & & $4,05(2,30)$ & & $3,82(1,60)$ & & 3,57 & $(1,13)$ & \\
\hline 6 & $8,05(1,32)$ & NS & $7,20(1,61)$ & $<0,05$ & $6,20(1,94)$ & $<0,001$ & $5,05(2,12)$ & $<0,001$ & 4,33 & $(2,06)$ & $<0,001$ \\
\hline
\end{tabular}

NS $=$ No significativo. 
Tabla 4

Necesidad de utilizar otros broncodilatadores según grupos estudiados

Otro broncodilatador

\begin{tabular}{lrrr} 
Grupo & $\mathrm{n}$ & \% & $\mathrm{p}$ \\
\hline 1 & $2 / 20$ & 10 & $<0,001$ \\
2 & $11 / 20$ & 55 & \\
3 & $3 / 20$ & 15 & $<0,001$ \\
4 & $11 / 20$ & 55 & \\
5 & $3 / 20$ & 15 & $<0,001$ \\
6 & $10 / 20$ & 50 & \\
\hline
\end{tabular}

aceptaron y en ninguno fue preciso suspenderlo por rechazo severo.

\section{DISCUSION}

Muchos lactantes con síndrome bronquial obstructivo tendrán en el futuro asma bronquial. Por esta razón, durante muchos años se ha utilizado en ellos medicamentos broncodilatadores con resultados variables, en especial los beta. miméticos, cuya efectivjdad tendría relación con la edad del paciente, como uno de los factores más importantes ${ }^{1,2}$. Una experiencia realizada con anterioridad en casos similares reveló la utilidad del fenoterol por vía oral en niños mayores de 16 meses de edad ${ }^{3}$.
El tono broncodilatador del músculo liso de la vía respiratoria se debe, en gran parte, a impilsos parasimpáticos colinérgicos. El efecto broncodilatador de los agentes anticolinérgicos se produciría principalmente en las vías respiratorias centrales más grandes, mientras los estimulantes de los receptores beta-adrenérgicos actuarian principalmente en la vía aérea periférica, aunque esto aún se discute ${ }^{8,20-24}$. Los lactantes responderian mejor a los anticolinérgicos (bromuro de ipratropio) que a los $\beta$-adrenérgi$\cos ^{4,8,9,17,25,26}$

En nuestra experiencia, la nebulización de una combinación de fenoterol con ipratropio en lactantes con sindrome bronquial obstructivo redujo significativamente la severidad clínica, los dias de estada y la necesidad de usar otro broncodilatador. Esta respuesta estuvo relacionada sólo con el uso del medicamento y no con la edad, sexo, estado de la nutrición u otros fac. tores.

La respuesta favorable fue independiente de la dosis. Esto subraya la importancia del efecto local del medicamento $y$ concuerda con otras experiencias en que la absorción de este tipo de medicamentos es uniforme $y$ prolongada en la mucosa respiratoria ${ }^{10,11,14,15}$. Sin embargo, al aumentar el intervalo entre las dosis a 12 horas, la respuesta fue menos favorable y sólo comparable a la de los otros grupos desde el segundo día de tratamiento. Posiblemente este hecho guarda relación con el número de nebuliza. ciones que recibieron los pacientes.

La tolerancia a la combinación empleada fue muy buena y no existieron efectos secundarios a las dosis usadas, lo que implica una gran seguridad en su uso ${ }^{5,7,17,13}$.

Tabla 5

Frecuencia cardíaca según grupos estudiados y dias de tratamiento

\begin{tabular}{|c|c|c|c|c|c|c|c|c|}
\hline \multirow{2}{*}{ Grupos } & \multicolumn{2}{|c|}{ Ingreso } & \multicolumn{2}{|c|}{ Día 1} & \multicolumn{2}{|c|}{ Día 2} & \multicolumn{2}{|c|}{ Día 3} \\
\hline & cpm & $\overline{\mathbf{x}} \quad(\mathrm{DE})$ & cpnl & $\bar{x}(\mathrm{DE})$ & cpm & $\overline{\mathbf{x}}$ (DE) & cpm & $\overline{\mathrm{x}} \quad(\mathrm{DE})$ \\
\hline 1 & 150,6 & $(6,19)$ & 144,9 & $(6,30)$ & 137,1 & $(8,10)$ & 135,8 & $(10,80)$ \\
\hline 2 & 144,6 & $(34,67)$ & 141,3 & $(33,80)$ & 137,8 & $(32,80)$ & 139,6 & $(5,90)$ \\
\hline 3 & 151,8 & $(5,90)$ & 144,7 & $(6,00)$ & 136,3 & $(9,30)$ & 132,8 & $(8,50)$ \\
\hline 4 & 142,8 & $(34,20)$ & 140,9 & $(33,50)$ & 138,0 & $(32,90)$ & 143,8 & $(6,10)$ \\
\hline 5 & 155,0 & $(6,63)$ & 143,6 & $(9,50)$ & 131,8 & $(32,00)$ & 138,1 & $(5,80)$ \\
\hline 6 & 151,1 & $(5,59)$ & 149,4 & $(5,00)$ & 145,7 & $(5.90)$ & 141,1 & $(6,60)$ \\
\hline $\mathrm{p}$ & \multicolumn{2}{|c|}{$>0,05$} & \multicolumn{2}{|c|}{$>0,05$} & \multicolumn{2}{|c|}{$>0,05$} & \multicolumn{2}{|c|}{$>0,05$} \\
\hline
\end{tabular}


El tratamiento con fenoterol e ipratropio en nebulizacjón, en lactantes con sindrome bronquial obstructivo, representa una posibilidad terapéutica altamente efectiva.

\section{RESUMEN}

Se realizó estudio prospectivo doble ciego en 120 lactantes con sindrome bronquial obstructivo agudo. La mitad de ellos fue tratada con nebulizaciones de fenoterol-ipratropio en 3 dosis diferentes y los restantes con solución de cloruro de sodio $9 \%$. Su estudio demostró una diferencia estadísticamente significativa $(\mathrm{p}<0,001)$ a favor de los grupos que recibieron fenoterol-ipratropio al evaluarlos mediante puntuación de la severidad de su obstrucción, dias de hospitalización y necesidad de recurrir a otro(s) broncodilatador(es). No se encontraron diferencias significativas $(\mathrm{p}>0,05)$ al comparar entre ellos los grupos que recibieron fenoterol-ipratropio en dosis diferentes. La combinación fenoterol-ipratropio presenta un efecto broncodilatador muy favorable en este grupo de pacientes y constituye una posibilidad terapéutica efectjva en lactantes afectados por síntomas bronquiales obstructivos agudos.

(Palabras clave: broncodilatadores, enfermedad bronquial obstructiva, lactantes, fenoterol, ipratropio.)

\section{REFERENCIAS}

1. Lenney $W$., Milner $A$. : Alpha and beta adrenergic stimulants in bronchiolitis and wheezy bronchitis in children under 18 months of age. Arch Dis Child 1978; $\$ 39$ : 707-709.

2. Lenney W., Milner $A .:$ At what age to bronchodilator drugs work? Atch Dis Child 1978; 53: $502-535$.

3. Calvo $\boldsymbol{M}$., Ide $A$,: Broncodilatadores en bronquitis obstructiva del lactante. Rev Chil Pediatt 1981; 52: $6: 476-480$.

4. Hodges I., Groggins R., Milner A., Stokes $G_{*}$ : Bronchodilator effect of inhaled ipratropium bromide in wheezy toddlers. Arch Dis Child 1981; 56: $729-731$.

5. Ruffin $R$ : A comparison of the bronchodilator activity of Sch 1000 and Salbutamol. J Allergy Clin Immunol 1977; 59: 136-141.

6. Storms $W$ : Aerosol Sch 1000: an anticolinergic bronchodilator. Am Rev Respì Dis 1975; 111: 419-424.

7. Stamforth $J$., Lewis $R$, Tattersfield A.: Dosage and delivery of nebulised $\beta$-agonist in hospital. Thorax 1983; 38: 751.754.

8. Gross $N$.; Farmacoterapia bromuro de iprattopio. N Engl J Med 1988; 319: 486-494.
9. Stokes G., Milner A., Hodges 1., Henry R., Mo Elphick $M:$ Nebulised therapy in acute severe bronchiolitis in infancy. Arch Dis Child 1983 58: $279-283$.

10. Fleischer $W .:$ Aerosoles y dosificadores. Técnicas y sistemas de apljcacjón. Periskop Magazin, 1983 13: $1-4$.

11. Newman S., Pavia $D$., Morien F., Sheahan $N$., Clarke $S$. : Deposition of pressurised aerosols in the human respiratory tract. Thorax 1981;36: 52-55.

12. Swift D.: Aerosols and humidity therapy. Am Rev Respix Dis 1980; 122: 71-77.

13. Willer $W$ : Aerosol therapy in acute and chronic tespiratory disease. Arch Intern Med 1973; 131: 148-155.

14. Clay M., Demetri P., Newman S., Clarke S.: Factors influencing the size distribution of aerosols from jet nebulisers. Thorax 1983; 38: 755-759.

15. Lewis $G$, Lewis $R_{n}$ : The place of nebulisers in chilhood asthma. Maternal and Child Health 1984 ; 9: $34-40$.

16. Lewis R., Cushley $M$., Fleming J., Tattersfield A.: Is a nebuliser less efficient than a metered dose inhaler, and do pear-shaped extension tubes work? Am Rev Respir Dis 1982; 125: 94-98.

17. Anónimo: Nebulizadores para el tratamiento del asma. Traducción de: Herxheimer A. (ed.) Drug and Ther Bull Dec 28, 1987; 25 (26) por Carta Médica, junio 1988. Publicación del Ministerio de Salud de Chile.

18. Berodual: Solución para inhalar. Basic Prescribing Information. Boehringer Ingelheim, International GmbH. Alemania, 1985; 1.6.

19. Tal A., Bayilski Ch., Yohai D., Bearmon J., Gorodischer R., Moses $S$. .: Dexamethasone and Salbuta$\mathrm{mol}$ in the treatment of acute wheezing in infants. Pediatrics, 1983; 71-1, 13-18.

20. Richordson J.B.: Nerve supply to the lungs. Am Rev Respit Dis 1979; 119: 785-802.

21. Bames P., Basbaum C., Nael J.: Autoradiographic localization of autonomic receptors in airway smooth muscle: matked differences between large and small airways. Am Rev Respir Dis 1983; 127: 758-762.

22. Hensley M., Cain C., Mc Fadden E., Ingram $R$. Distribution of bronclrodilatation in normal subjects: beta-agonist versus atropine. J Appl Physiol 1978: 45: 778-782

23. Ashutosh K., Mead G., Dickey J., Jr, Berman P., Kuppinger $\boldsymbol{M}$.: Density dependence of expiratory flow and btonchodilator response in asthma. Chest, 1980;77: 68-75.

24. Partridge $M$., Saunders $K$.: Site of action of ipratro pium bromide and clinical and physiological de terminants of response in patients with asthma. Thorax, 1981; 36: 530-533.

25. Chagman K., Smith D., Rebuck A., Leenen F.: Hemodynamic effects of inhaled Ipratropium bromide, alone and combined with and inhaled beta-2-agonist. Am Rev Respir Dis 1985; 132: 845-847.

26. Ullah $M$., Newman $G$., Saunders $K$.: Influence of age on response to ipratropium and Salbutamol in asthma. Thorax; $1981 ; 36: 523-529$. 